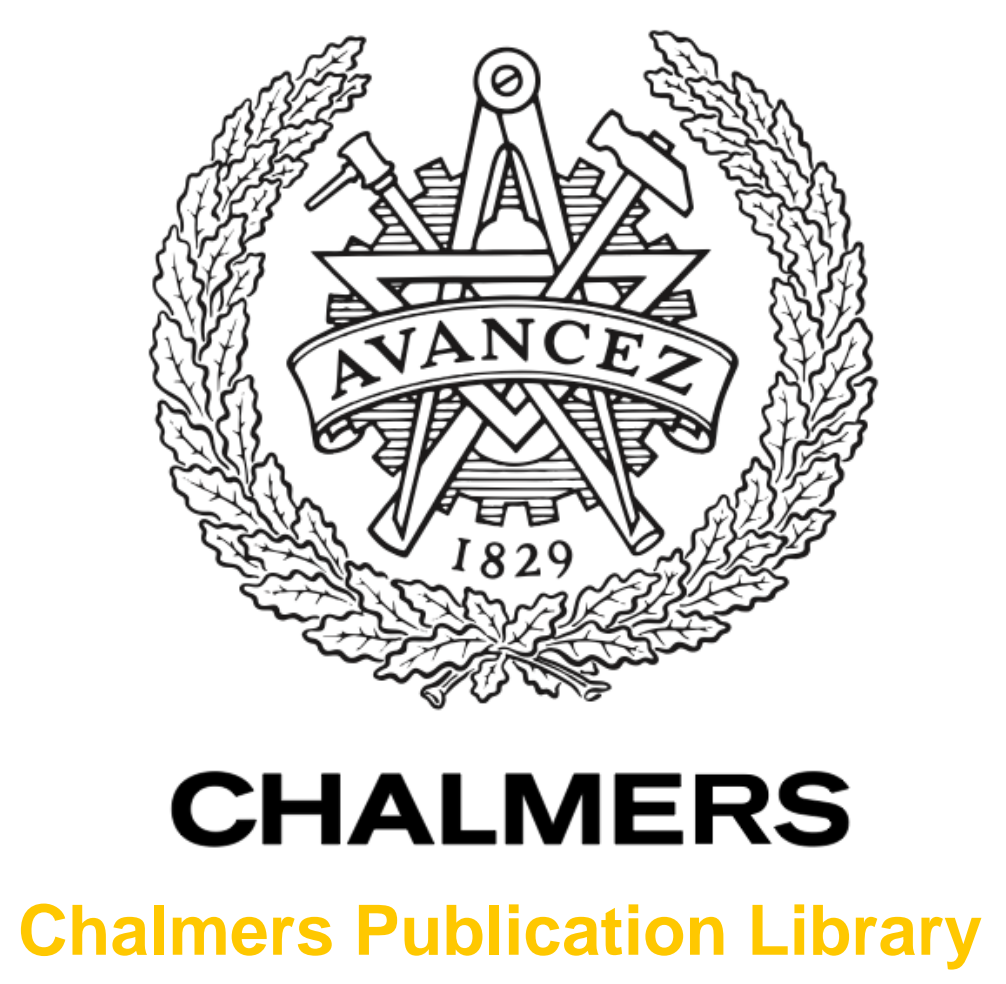

WDM channel capacity and its dependence on multichannel adaptation models

This document has been downloaded from Chalmers Publication Library (CPL). It is the author's version of a work that was accepted for publication in:

2013 Optical Fiber Communication Conference and Exposition and the National Fiber Optic Engineers Conference, OFC/NFOEC [Invited]

Citation for the published paper:

Agrell, E. ; Karlsson, M. (2013) "WDM channel capacity and its dependence on multichannel adaptation models". 2013 Optical Fiber Communication Conference and

Exposition and the National Fiber Optic Engineers Conference, OFC/NFOEC [Invited]

Downloaded from: http://publications.lib.chalmers.se/publication/186983

Notice: Changes introduced as a result of publishing processes such as copy-editing and formatting may not be reflected in this document. For a definitive version of this work, please refer to the published source. Please note that access to the published version might require a subscription. 


\title{
WDM Channel Capacity and its Dependence on Multichannel Adaptation Models
}

\author{
Erik Agrell(1) and Magnus Karlsson(2) \\ (1) Dept. of Signals and Systems, (2) Dept. of Microtechnology and Nanoscience, \\ Chalmers University of Technology, SE-412 96 Göteborg, Sweden \\ email:agrell@chalmers.se
}

\begin{abstract}
Optical multichannel systems are often characterized by the channel capacity of a single channel, assuming a certain adaptation behavior of the other channels. We investigate some common adaptation models, which lead to dramatically different capacities.
\end{abstract}

OCIS codes: (060.4080) Modulation, (060.4510) Optical communications

\section{Introduction}

The channel capacity $C$ is the largest possible spectral efficiency of a given communication channel, for any combination of modulation format and error-correcting code. The maximum spectral efficiency over all codes, for a given modulation format, is proportional to the mutual information between the input and output [1], [2, Ch. 2, 7]. The channel capacity is thus theoretically obtained by maximizing the mutual information over all modulation formats (input distributions), which unfortunately is very difficult to carry out in general. Therefore, the channel capacity of most nonlinear channels is unknown, but upper and lower bounds exist.

In optical fiber channels, the fiber nonlinearity is known to limit the capacity [3-6]. However, this does not prohibit the capacity to grow monotonically with signal power [7] for all so-called static channel models. The statistics of static channel models are invariant to the input statistics, which is the case for practically all channel models studied in information theory $[1,2]$ and also for the class of optical fibers described by the nonlinear Schrödinger equation with additive inline amplifier noise.

The capacity in a multiuser system, such as a set of wavelength-division multiplexed (WDM) subchannels, where a number of interfering users will limit the capacity of the channel under study, is more subtle to analyze. The governing paradigm in optical multiuser communications is to analyze it from the viewpoint of one single user in the system, say user 1, assuming that the other users are outside our control. In other words, the quantity of interest is $C=\max I\left(X_{1} ; Y_{1}\right)$, where $I\left(X_{1} ; Y_{1}\right)$ denotes the mutual information between the input $X_{1}$ and output $Y_{1}$ of subchannel 1 , and the maximization is over all possible input distributions (modulation formats) $f_{X_{1}}$. This is in contrast to wireless multiuser systems, where the transmitters are typically designed jointly (but possibly operated separately), and the relevant capacity measure is a multidimensional object, the capacity region [2, Ch. 15], which gives the set of achievable spectral efficiencies over all subchannels.

In order to describe a multiuser system as a single-user channel model $X_{1} \rightarrow Y_{1}$, two models are needed, as illustrated in Fig. 1: not only a discrete-time multiuser channel model, which gives the outputs $Y_{1}, \ldots, Y_{M}$ as a function of the inputs $X_{1}, \ldots, X_{M}$, but also a behavioral model (or adaptation model), which relates the interference distributions $f_{X_{2}}, \ldots, f_{X_{M}}$ to the primary input distribution $f_{X_{1}}$. Obviously, $f_{X_{1}}$ needs to be optimized for a certain channel in order to attain the channel capacity, but how shall the other users (interferers) behave during this optimization process? Will they be passive, or are they allowed to adapt their signaling power and/or modulation format to the power and/or modulation format of user 1 ? We will see that the choice of behavioral model for the interferers as transmitter 1 changes its operation has a profound impact on the capacity of channel 1.

\section{System model}

As an illustrative example, we consider a simplified model of an optical link with three WDM channels, corresponding to three equispaced wavelengths, limited by four-wave mixing. Under some simplifying assumptions (e.g., weak nonlinearity and negligible dispersion), the complex discrete-time output signals $Y_{i}$ for $i=1,2,3$ are given by a nonlinear channel model according to

$$
Y_{1}=X_{1}+i \varepsilon X_{2}^{2} X_{3}^{*}+N_{1} \quad Y_{2}=X_{2}+2 i \varepsilon X_{1} X_{2}^{*} X_{3}+N_{2} \quad Y_{3}=X_{3}+i \varepsilon X_{1}^{*} X_{2}^{2}+N_{3}
$$

where $X_{i}$ are independent, complex channel inputs and $N_{i}$ are independent, complex, circularly symmetric, white Gaussian noise signals, each with zero mean and equal variance. In an $n$-stage amplified link, the noise variance (power) equals $P_{\text {ase }}=n n_{\mathrm{sp}}(G-1) h v B$, where $n_{\mathrm{sp}}$ is the spontaneous emission factor, $h v$ the photon energy, $G$ the 


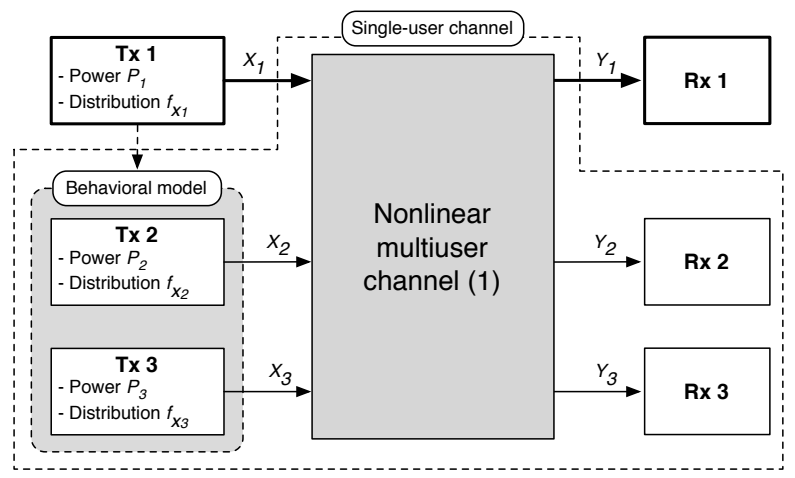

Fig. 1: A combination of a behavioral model for all users but one and a multiuser channel model yields a single-user channel model. Transmitter and receiver are abbreviated $\mathrm{Tx}$ and Rx, respectively.

amplifier gain, and $B$ the signal bandwidth. The constant in (1) is $\varepsilon=n \gamma L_{\text {eff }}$, where $\gamma$ is the fiber nonlinear coefficient and $L_{\text {eff }}$ the effective nonlinear amplifier span length. A similar model (but generalized to more wavelengths) was derived in [8]. We use this model for its relative simplicity and illustrative purposes, rather than aiming for an accurate model of a physical transmission scenario. In this paper, $n_{\mathrm{sp}}=2, G=1000, n=16, B=40 \mathrm{GHz}, h v=0.128$ aJ, $\gamma=1.6 \mathrm{~W}^{-1} \mathrm{~km}^{-1}$, and $L_{\text {eff }}=24 \mathrm{~km}$, which gives $P_{\text {ase }}=0.16 \mathrm{~mW}$ and $\varepsilon=610 \mathrm{~W}^{-1}$. The signal powers are denoted by $P_{i}=\mathbb{E}\left[\left|X_{i}\right|^{2}\right]=\int|x|^{2} f_{X_{i}}(x) d x$.

\section{Behavioral models in multiuser communications}

Whenever a multiuser system is characterized by means of a single-user channel capacity, the results are connected to a certain behavioral model, as discussed above. We study three fundamentally different classes of behavioral models: (a) fixed interference, (b) adaptive interference power, and (c) adaptive interference distribution.

Model (a) is the simplest one: The interference distributions $f_{X_{2}}, \ldots, f_{X_{M}}$ remain the same regardless of $f_{X_{1}}$. The dashed arrow from Tx 1 in Fig. 1 does not exist in this case.

Model (b) means that all users transmit with the same power $P_{1}=P_{2}=P_{3}$, but not necessarily the same distributions. The interference distributions $f_{X_{2}}, \ldots, f_{X_{M}}$ are fixed apart from a scale factor, which depends on $P_{1}$. If one first derives a single-user channel model $X_{1} \rightarrow Y_{1}$ assuming that all transmitters use the same modulation format $[9,10]$ and then studies the capacity of this single-user channel model, as in [11], then the underlying behavioral model is automatically in class (b), because one distribution is optimized and the others are not.

Model (c), finally, means that all users transmit with the same distribution and the same power, $f_{X_{1}}=f_{X_{2}}=f_{X_{3}}$. This model was used in $[6,12,13]$.

Other studies of WDM systems in terms of a single-user channel capacity are $[3,4,14]$. They clearly assume the same power on all wavelengths, but it is not clear to us whether these works assume behavioral model (b) or (c). The capacity may be very different in the two cases, as will be demonstrated in the next section.

\section{Results}

Fig. 2 (a)-(c) show the single-user channel capacity $C\left(P_{1}\right)=\max I\left(X_{1} ; Y_{1}\right)$ of the multiuser channel in Sec. 2, where the maximization is over all distributions $f_{X_{1}}$ with power $P_{1}$, combined with the three behavioral models in Sec. 3. For models (a) and (b), the interference distributions $f_{X_{2}}$ and $f_{X_{3}}$ are either uniform over a QPSK constellation or Gaussian. As usual, we are unable to calculate the capacity exactly, but we can sandwich it between upper and lower bounds (shaded areas). No approximations are involved in the derivations of these bounds, apart from numerical integration.

The lower bounds (solid) in Fig. 2 are obtained as follows. With models (a) and (b) and QPSK interference (green), the lower bounds equals $I\left(X_{1} ; Y_{1}\right)$ for a Gaussian input $X_{1}$, calculated by numerical integration. The Mitra-Stark lower bound $[4,14]$ was used with models (a) and (b) and Gaussian interference. Finally, $I\left(X_{1} ; Y_{1}\right)$ with $f_{X_{1}}$ being uniform over a multilevel phase-shift keying constellation provides the lower bound for model (c), where the number of levels is optimized for each power. These lower bounds are certainly not tight. E.g., so-called satellite constellations would be able to improve the bounds in some regions [15].

As an upper bound (dashed), the conditional mutual information $\max I\left(X_{1} ; Y_{1} \mid X_{2}, X_{3}\right)$, which represents a Gaussian channel, is always valid. It is used in Fig. 2 (a) and (b) with QPSK interference and also in Fig. 2 (c). For models (a) and (b) with Gaussian interference, the bound can be sharpened to $\max I\left(X_{1} ; Y_{1} \mid X_{2}\right)$, which also represents a Gaussian 

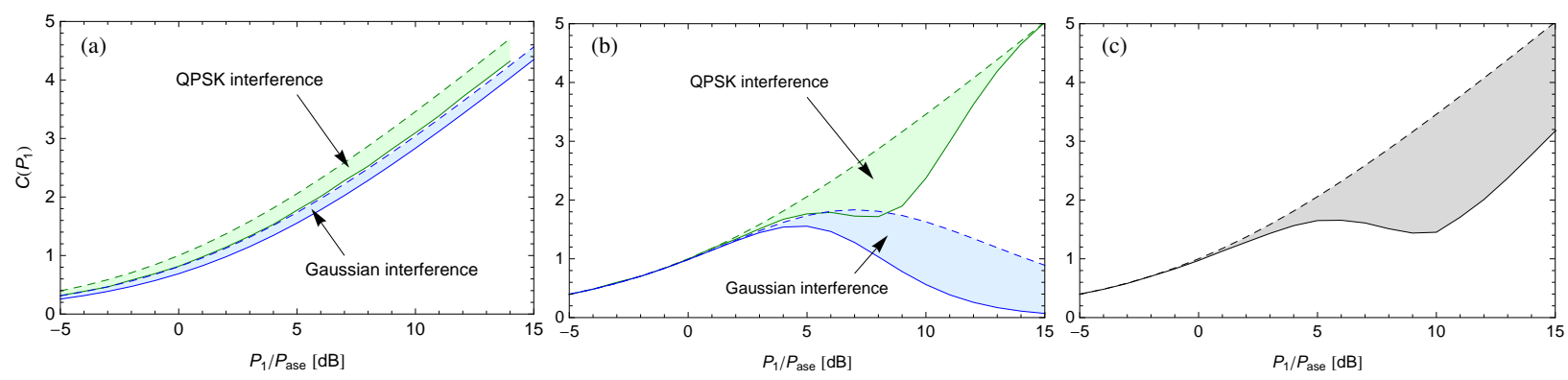

Fig. 2: The channel capacity $C\left(P_{1}\right)$ of user 1 in a WDM system, with the three behavioral models in Sec. 3, as a function of the transmit power $P_{1}$. Shaded regions indicate the amount of uncertainty. The interference power is fixed, $P_{2} / P_{\text {ase }}=P_{3} / P_{\text {ase }}=5 \mathrm{~dB}$, in model (a).

channel in this case. Analytical expressions for all these bounds have been derived, but they are omitted for space constraints.

\section{Conclusion and discussion}

As seen in Fig. 2, the channel capacity increases to infinity as the transmitted power increases, for behavioral models (a) and (c). With model (b), however, the outcome depends crucially on the interference distributions; the capacity may increase indefinitely, as with the other behavioral models, or it may decrease to zero. Although these results were here demonstrated only for an over-simplified memoryless WDM model (1), the same trends have been proven for all static multiuser channel models [7], including channels with (finite) memory: The channel capacity with behavioral models (a) and (c) is always increasing, which is not the case with model (b). The irregular behavior of model (b) arises because it converts any multiuser channel, static or dynamic, into a dynamic single-user model (dashed region in Fig. 1) [7].

We emphasize that whenever a single-user channel model is derived for a multiuser system, there is always an underlying behavioral model involved, even if not explicitly stated. Despite their significance, behavioral models have not yet received much attention in optical communications. Our recommendation to everyone working with the capacity of such single-user channel models is to clearly state and justify the behavioral model, because it has a profound impact on the end results.

We wish to acknowledge inspiring discussions with colleagues within the Chalmers FORCE center.

\section{References}

1. C. E. Shannon, "A mathematical theory of communication," Bell System Technical Journal, vol. 27, pp. 379-423, 623-656, July, Oct. 1948.

2. T. M. Cover and J. A. Thomas, Elements of Information Theory, 2nd ed. Hoboken, NJ: Wiley, 2006.

3. A. Splett, C. Kurtzke, and K. Petermann, "Ultimate transmission capacity of amplified optical fiber communication systems taking into account fiber nonlinearities," in Proc. European Conference on Optical Communication (ECOC), Sept. 1993, pp. 41-44.

4. P. P. Mitra and J. B. Stark, "Nonlinear limits to the information capacity of optical fibre communications," Nature, vol. 411, pp. 1027-1030, June 2001.

5. K. S. Turitsyn, S. A. Derevyanko, I. V. Yurkevich, and S. K. Turitsyn, "Information capacity of optical fiber channels with zero average dispersion," Physical Review Letters, vol. 91, no. 20, pp. 203 901-1-4, Nov. 2003.

6. R.-J. Essiambre, G. Kramer, P. J. Winzer, G. J. Foschini, and B. Goebel, "Capacity limits of optical fiber networks," J. Lightw. Technol., vol. 28, no. 4, pp. 662-701, Feb. 2010.

7. E. Agrell, "On monotonic capacity-cost functions," 2012, preprint. [Online]. Available: http://arxiv.org/abs/1209.2820

8. M. Eiselt, "Limits on WDM systems due to four-wave mixing: A statistical approach," J. Lightw. Technol., vol. 17, no. 11, pp. 2261-2267, Nov. 1999.

9. P. Poggiolini, A. Carena, V. Curri, G. Bosco, and F. Forghieri, "Analytical modeling of nonlinear propagation in uncompensated optical transmission links," IEEE Photon. Technol. Lett., vol. 23, no. 11, pp. 742-744, June 2011.

10. A. Carena, V. Curri, G. Bosco, P. Poggiolini, and F. Forghieri, "Modeling of the impact of nonlinear propagation effects in uncompensated optical coherent transmission links," J. Lightw. Technol., vol. 30, no. 10, pp. 1524-1539, May 2012.

11. G. Bosco, P. Poggiolini, A. Carena, V. Curri, and F. Forghieri, "Analytical results on channel capacity in uncompensated optical links with coherent detection," Optics Express, vol. 19, no. 26, pp. B440-B449, Dec. 2011.

12. R.-J. Essiambre, G. J. Foschini, G. Kramer, and P. J. Winzer, "Capacity limits of information transport in fiber-optic networks," Physical Review Letters, vol. 101, no. 16, pp. 163 901-1-4, Oct. 2008.

13. T. Freckmann, R.-J. Essiambre, P. J. Winzer, G. J. Foschini, and G. Kramer, "Fiber capacity limits with optimized ring constellations," IEEE Photon. Technol. Lett., vol. 21, no. 20, pp. 1496-1498, Oct. 2009.

14. L. G. L. Wegener, M. L. Povinelli, A. G. Green, P. P. Mitra, J. B. Stark, and P. B. Littlewood, "The effect of propagation nonlinearities on the information capacity of WDM optical fiber systems: Cross-phase modulation and four-wave mixing," Physica D: Nonlinear Phenomena, vol. 189, no. 1-2, pp. 81-99, Feb. 2004.

15. E. Agrell and M. Karlsson, "Satellite constellations: Towards the nonlinear channel capacity," in Proc. IEEE Photon. Conf. (IPC), Burlingame, CA, Sept. 2012. 